\title{
Operating a Telescope Larger than the Earth: How to Schedule the HALCA Space VLBI Mission
}

\section{L. Meier}

Jet Propulsion Laboratory, Caltech, Pasadena, CA 91109, U.S.A.

\begin{abstract}
We outline the HALCA Space VLBI mission scheduling process, with special emphasis placed on software that decides when to observe the proposed sources and how to ensure that the highest quality science is obtained. This information will be useful to the many principal investigators whose very high resolution VLBI observations are being supported by the mission.
\end{abstract}

The scientific scheduling of even ground-based radio astronomical observations is not always an easy task. This is especially true of Very Long Baseline Interferometry (VLBI) observations which sometimes involve ad hoc collections of ground radio telescope (GRT) facilities around the world, each with its own separate scientific agendae, operation policies, and maintenance and personnel schedules. Similarly, the scheduling of space astronomy observations (e.g., with the Hubble Space Telescope or the Compton Gamma Ray Observatory) is also difficult, due to a variety of spacecraft pointing, solar power, radiation, and telecommunication constraints that often require the accumulation of data over several orbits in disjointed pieces. The scheduling of Space VLBI experiments, however, is significantly more complex than either of these, not only because it combines the problems of both in an attempt to create a telescope larger than the earth, but also because it imposes additional constraints not associated with either ground or space astronomy. Those new conditions are 1) the spacecraft must be in constant contact with a ground tracking station (GTS) in order to uplink a stable local oscillator signal and downlink the several terabits of data generated per observation (Hirosawa \& Hirabayashi 1996; Hirabayashi, these Proceedings, p. 11); 2) the observed radio source must lie fairly close to the projected orbit normal in order to obtain full two-dimensional coverage of the aperture plane (Murphy et al. 1994); and 3) the source sky position must be lie $70^{\circ}$ or more from the sun in order that the large radio antenna does not shade the solar panels and reduce on-board power levels (Kobayashi 1991). The first constraint adds yet another network of antennas to be scheduled, while the second two impose severe limits on the dates when a given radio source can be observed, due to the slow movement of the orbit normal and sun across the celestial sphere.

In order to solve the space VLBI scheduling problem, we have created a piece of software, called SVLBSCHED, which performs both long-term and shortterm scheduling tasks. For long-term scheduling we use a "limited-resource" algorithm which orders the observations in scientific priority (determined by the independent Science Review Committee) and schedules from the top down until a lack of resources prevents further scheduling. Observations with equal priority are further ordered according to increasing number of good observing days available. "Good observing days" are determined mainly by the quality of $(u, v)$-coverage attainable (two-dimensional extent in $u$ and $v$ and the number of data points, weighted by the number of GRTs on source and the RMS spread in ground-ground baseline length). Version 1 of this software (Meier 1994) 
considered both the VSOP/HALCA and Radioastron missions simultaneously, automatically generated an 18-month schedule of observations from an input list of sources, and assigned ad hoc arrays of GRTs for observing. It was used to determine the requests for observing time submitted to the GRTs. Version 2 ( $c$. 1994-7) added features needed for actual long-term scheduling: consideration of organized VLBI arrays (VLBA, EVN, SHEVE, etc.), the non-compatibility of VLBI recording systems, correlator and tape copy machine constraints, multipleobservation (monitoring) experiments, and a user interface for manual manipulation of the schedule. Version 3 , currently in use by the Japanese HALCA mission, addresses short-term scheduling as well, generating a detailed list of commands (the Global Space Telescope Schedule or GSTS file) for all mission elements either automatically from simple input lists of sources or from detailed instructions from the scheduler. One of its most important tasks is to plan the spacecraft slews, determine the proper post-slew spacecraft orientation, and generate the pointing calibration sequence using the (radiation-sensitive) star trackers.

The entire HALCA scheduling process proceeds as follows. Input files are a source catalog, a catalog of the mission elements' physical capabilities, an availability schedule for all elements, a list of experiments to be done based on the scientific proposals, and an optional trial schedule for some or all of the proposed experiments (generated by software written by $\mathrm{E}$. Fomalont of NRAO and the Japanese Institute of Space and Astronautical Sciences [ISAS]). SVLBSCHED creates a GSTS file for the observations, which is then checked and corrected if necessary for recent orbital information, current spacecraft and tracking station conditions, etc. by software written by ISAS's Y. Murata. For the spacecraft and GTSs, further software written by R. Okayasu of ISAS generates a Space Radio telescope Schedule (SRS) file for the current week of operation. This file is similar in format to the GSTS file, but with some commands expanded into detailed sequences that execute slews, source acquisition, power operations, etc.. For the GRTs, software written by P. Edwards of ISAS converts the GSTS file into KEYIN input files for the program NRAO SCHED, which is then used to create the DRUDG, VEX, or NRAO card files that drive the various GRTs. Finally, the preliminary schedule is sent to all participating mission elements for review.

Acknowledgments. Part of this research was carried out at the Jet Propulsion Laboratory, California Institute of Technology, under contract to NASA.

\section{References}

Hirosawa, H. \& Hirabayashi, H. 1996. Space Technology, 16, 161-167.

Kobayashi, H. 1991. in Frontiers of VLBI, eds. H. Hirabayashi, M. Inoue, \& H. Kobayashi (Tokyo: Universal Academy Press), 95-98.

Meier, D. L. 1994. in VLBI Technology: Progress and Future Observational Possibilities, eds. T. Sasao, S. Manabe, O. Kameya, \& M. Inoue (Tokyo: Terra Scientific Publishing), $39-43$.

Murphy, D. W., et al. 1994. in VLBI Technology: Progress and Future Observational Possibilities, eds. T. Sasao, S. Manabe, O. Kameya, \& M. Inoue (Tokyo: Terra Scientific Publishing), 34-38. 